\title{
Características de la construcción curricular de las carreras universitarias de Contabilidad en Chile
}

\section{Features of the curricular construction of accounting undergraduate programs in Chile}

\author{
Ariel La Paz Lillo', Juan Carlos Armijos Armijos²
}

\begin{abstract}
RESUMEN
La presente investigación analiza las carreras de contabilidad en cuanto a las características principales del diseño y construcción de los perfiles de egreso. Busca, además, identificar los aspectos diferenciadores en la construcción y creación de las distintas denominaciones que se le ha dado a la carrera en Chile. El estudio propone responder ¿Cuáles son las principales características de la formación del contador auditor declarada por las universidades chilenas? La investigación utiliza un método cualitativo para contextualizar información disponible públicamente de manera ordenada y sistemática, presentando un marco ontológico que permite visibilizar de manera integral los elementos y componentes disponibles en los procesos de diseño y construcción curricular, así como analizar los constructos académicos implícitos en las carreras universitarias de contabilidad que actualmente se ofrecen en Chile. El marco ontológico conceptualiza los dominios de desempeño profesional de manera exhaustiva, revelando a través de la organización y estandarización de términos en taxonomías, las posibles combinaciones que finalmente se expresan en perfiles de egreso y mallas curriculares. Los resultados del estudio muestran un importante nivel de heterogeneidad y diversidad en las competencias que los egresados son formados. En cuanto a los Productos que un egresado puede ofrecer al mercado sobresalen los Servicios; en materia de Competencias Transversales la Ética; en las Competencias Técnicas Asesorar; y el principal objetivo educativo es Crear. En cuanto al dominio de materias que dispone el egresado sobresalen la Contabilidad, y la Administración y Operaciones y Auditoría.
\end{abstract}

Palabras clave: contabilidad, diseño curricular, educación superior, mallas curriculares, perfiles de egreso.

Recepción: 20/08/2020. Aprobación: 29/12/2020

1 Universidad de Chile, Facultad de Economía y Negocios, Santiago de Chile, Chile, lapaz@fen.uchile.cl

2 Universidad Santo Tomás, Facultad de Economía y Negocios, Santiago de Chile, Chile, jarmijos@santotomas.cl 


\begin{abstract}
The study analyzes accounting undergraduate programs in the main characteristics of their design and construction of graduation profile. It also aims to identify the differentiating factors in the definition of the different denominations given to these programs in Chile. The research seeks to answer: What are the main characteristics of the training of the auditor accountant publicized by the Chilean universities? The research utilizes a qualitative method to contextualize and organize the publicly available information systematically, introducing an ontological framework, which allows to entirely visualize the elements and components used in curricular design and construction, as well as to analyze the academic constructs implicit in the current academic offer. The ontological framework conceptualizes the professional performance domains exhaustively, revealing the potential combinations that shape the graduation profile and curricular program in taxonomies. Study results show a heterogeneity and diversity in the abilities that the graduates are trained to perform. Regarding the products that a graduate can supply to the labor market outstand the accounting services; while in the complementary areas, ethics is a major competence. In technical abilities, the capacity to advise, and the educational objective to create are important trends. Main domains for the education and training of the graduates are accounting, administration and operations management, and auditing.
\end{abstract}

Keywords: accounting, curriculum, curriculum design, higher education, profiles of graduates.

\title{
INTRODUCCIÓN
}

Las carreras de contabilidad son de interés social por su impacto en la formulación y práctica de normativa financiera, legal, tributaria, administrativa y de áreas afines que afectan e impactan el desempeño y el orden económico de un país (Wyman, 1990). Ésta es la disciplina que forma al capital humano avanzado responsable en gran medida por el registro sistemático y el análisis de las transacciones que ocurren en la economía. La formación de contadores y especialistas en las subdisciplinas contables es por tanto un factor crítico para el desarrollo de las naciones por su función en la promoción de la transparencia, presentación de información al mercado, integración corporativa en una economía globalizada y la actualización permanente de la disciplina (Wyman, 1990). Luego, es importante reconocer cuáles son las características principales en la formación de contadores, por ejemplo, para identificar alguna tendencia en la formación y potencialmente diferencias que se puedan evidenciar entre las varias denominaciones de grados académicos y títulos profesionales que se encuentra en la oferta universitaria chilena.

La Federación Internacional de Contadores (International Federation of Accountants / IFAC), publicó las Normas Internacionales de Formación en Contaduría, cuyo objetivo principal, es servir al interés público y fortalecer la profesión contable mediante: El desarrollo de estándares de alta calidad; Promover la adopción e implementación de esos estándares; Apoyar en el fortalecimiento de la capacidad de las organizaciones contables profesionales; y hablar sobre temas de interés público (IFAC, 2019). Para dar cumplimiento con dicha misión, el Consejo de IFAC ha establecido el Consejo de Normas Internacionales de Formación en Contaduría (IAESB) quien ejerce el papel de organismo emisor de normas independiente, el cual "desarrolla normas de infor- 
mación, orientación y documentos de información para su uso por los miembros de la IFAC, y de partes interesadas, tales como: universidades, empleadores, contadores" (IFAC, 2019), etc.

Con el fin de asegurar la calidad académica en la formación profesional, las Instituciones de Educación Superior (IES) desarrollan procesos de creación, modificación, revisión, o innovación curricular atendiendo a los parámetros de calidad educativa, nuevos modelos y métodos de enseñanza y aprendizaje, demandas del medio, y a impulsos de competencia y posicionamiento entre las comunidades académicas (López, 2017). El proceso de diseño y construcción curricular es un evento cada día más recurrente en las IES. Se trata de un proceso minucioso, que requiere de la precisión que ofrece un saber experto o especializado, como de un carácter interdisciplinario. La creciente necesidad de revisar y mejorar los diseños y procesos curriculares responde en primer lugar a la transición hacia modelos de educación por competencias, y, en segundo lugar, a la evaluación, medición y acreditación de la calidad académica, y el establecimiento de procesos como parte de una cultura de mejora continua, vinculados también a mecanismos de acreditación vigentes y/o exigibles, de acuerdo con el modelo adoptado en cada país.

Para desarrollar los procesos relacionados al diseño curricular, las IES siguen principalmente dos procedimientos. El primero y más habitual, es apoyarse en expertos en diseño curricular y expertos disciplinares, en las definiciones provistas por organismos rectores facultados para habilitar carreras y programas, y organismos que evalúan y acreditan la calidad educativa de tercer nivel (Troncoso y Hawes, 2007). Tanto expertos como organismos rectores y evaluadores proveen bases que pueden entenderse como recomendaciones, estándares de cumplimiento o incluso restricciones para el diseño, construcción e implementación curricular en la formulación de perfiles de ingreso/egreso, definiciones de competencias profesionales, planes de estudio y estructuras curriculares con las que las que las IES formulan su oferta académica. En algunos países, estos procesos son estructurados por acuerdos generales, tales como marcos de cualificaciones (CEDEFOP, 2012; Ministerio de Educación de Colombia, 2017), pero en la mayoría de los países latinoamericanos los componentes sociales y políticos de cada institución llegan a sesgar los procesos de construcción curricular por visiones específicas en cuanto a oferta y demanda, o intereses particulares de los propios incumbentes que participan del diseño curricular. Chile no cuenta con un marco de cualificaciones integral que direccione procesos de diseño curricular, y el mejor avance disponible a la fecha es el que ha desarrollado la División de Educación Superior para el nivel Técnico Profesional.

La segunda práctica habitual de los procesos de innovación o diseño curricular es el benchmark, o comparación con programas de referencia, adaptándolos a la realidad local. Además, esta modalidad comprende o incluye la realización de consultas al medio y a la comunidad universitaria. Ambas prácticas están afectas a la injerencia de actores políticos, impactando la calidad del diseño curricular y los resultados en la formación. Consecuentemente, tales sesgos pueden llegar a afectar negativamente la coherencia interna de los programas, su alineamiento institucional, o la consistencia para responder a la demanda formativa del medio (López, 2016). Tales fuerzas producen distorsiones que se evidencian en diferencias nominales en las denominaciones de título profesional, materias o perfiles de egreso, y de ello se desprende la necesidad de contar con un proceso sistemático que facilite la identificación de áreas de interés para la especialización y diferenciación de los programas académicos por nivel (pre-post grado), por temática de aplicación profesional, o por el enfoque de la institución educativa, permitiendo la comparación entre 
programas. Un proceso sistemático facilitaría también la definición de mecanismos de homologación, internacionalización y movilidad de estudiantes y académicos asociados a los núcleos disciplinares.

Por su parte, los procesos socio-político-técnicos han producido en Chile 36 programas universitarios de pregrado en contabilidad con duración igual o superior a 8 semestres. Estos programas se encuentran bajo diferentes denominaciones, estructuras curriculares, perfiles de egreso, e intenciones formativas. Es también claro que cada IES desarrolla estos procesos académicos con esquemas de contratación de académicos y docentes, recursos de infraestructura física y tecnológica, o modalidades de estudio que difieren de manera importante, parte de lo cual se refleja en los años de acreditación obtenidos por aquellos programas que se sometieron a procesos de evaluación y acreditación de la calidad. El trabajo de (Armijos y La Paz, 2019) determina que, en un universo de 3.425 universidades latinoamericanas de habla hispana, 673 instituciones ofrecen la carrera, aunque bajo 39 denominaciones o títulos distintos, revelando que incluso en una profesión que se puede describir como tradicional, existe una variedad importante en las denominaciones, perfiles de egreso y planes curriculares. Esto plantea varias interrogantes que requieren ser explicadas. En primer lugar, es notorio que existe una diferenciación en las denominaciones, duración, y modalidad de enseñanza, traducido en similares denominaciones con distinta duración y composición curricular, o similar composición y duración, pero diferente denominación. Por otro lado, dada la diversidad en las denominaciones se tiende a enfatizar algunas áreas de especialización, por ejemplo: Tributación, Contabilidad de Gestión, Contabilidad y Finanzas, etc. mientras que otras IES prefieren una denominación genérica, como Contador Auditor, o Contador Público Auditor, por mencionar algunas.

Este estudio propone responder ¿Cuáles son las principales características de las carreras de contabilidad declaradas por las universidades chilenas? Responder esta pregunta resulta relevante ya que permitiría conocer, entre otros, los tipos de orientación formativa que existen de la carrera, así como explorar la relación existente entre las denominaciones y los perfiles de egreso y sus mallas curriculares. Para realizar esta labor, se presenta un marco ontológico diseñado para caracterizar la carrera, analizar los datos y reportar los resultados del estudio. El método de análisis ontológico ha sido aplicado en varias áreas y campos del conocimiento que presentan fenómenos complejos para su conceptualización y estudio.

\section{ESTADO DEL ARTE}

En la ciudad de Bolonia, en el año 1988 se estableció la Carta Magna de las Universidades Europeas que proclama principios básicos para una reforma en la Educación Superior. En 1998 fue reforzado en la Declaración de la Sorbona, y un año más tarde, 29 ministros de Educación europeos firmaron la declaración que da origen al Proceso de Bolonia que conforma el Espacio Europeo de Educación Superior. A través de esta declaración, se inició un proceso de convergencia que tenía como objetivo facilitar el intercambio de titulados y adaptar el contenido de los estudios universitarios a las demandas sociales, mejorando su calidad y competitividad a través de una mayor transparencia. Así en Europa, el Centro Europeo para el Desarrollo de la Formación Profesional (CEDEFOP) establece, diseña y recomienda marcos nacionales de cualificaciones (MNC) como herramientas e instrumentos para la transparencia y el cambio, definiendo que "los 
marcos nacionales de cualificaciones (MNC) se clasifican según un conjunto de niveles basados en los resultados del aprendizaje". Unificando los MNC, el Marco Europeo de Cualificaciones (MEC) permite comparar las cualificaciones entre los diferentes países del continente desde 2008, erigiéndose en un marco de referencia común que actúa como mecanismo de conversión entre distintos sistemas de cualificaciones y sus respectivos niveles, promoviendo el aprendizaje permanente, la coordinación y la movilidad (CEDEFOP, 2017).

A nivel mundial, los marcos de cualificaciones están siendo desarrollados en varios países. Un reporte de la Organización Internacional del Trabajo (OIT), menciona que para el año 2010, alrededor de 100 países, disponían o se encontraban en proceso de implementación de MNC, destacando que "La European Training Foundation (ETF), junto con UNESCO y CEDEFOP, han desarrollado un inventario online de MNC que abarca 42 países en Europa del Este, Asia Central, Sudeste y Sur, Balcanes, Turquía y Oceanía." (Zúñiga, 2018). En Latinoamérica, países como Colombia, Chile, Costa Rica, República Dominicana, y Perú, también se encuentran trabajando en el desarrollo e implementación de MNC. En el continente, el proyecto Tuning-América desarrollado en 2007, reflexiona sobre la educación superior. Tuning es una metodología internacional que busca identificar e intercambiar información y mejorar la cooperación entre las IES con vistas en el fomento de la calidad. Entre sus objetivos principales se encuentra el de contribuir con el desarrollo de titulaciones fácilmente comparables y comprensibles, que posibiliten ampliar la articulación entre los sistemas de educación superior de los países, posibilitando de este modo, la movilidad de los profesionales. Para ello es necesario promover el consenso académico a nivel regional sobre el modo de entender los títulos, desde el punto de vista de las competencias que los poseedores de ellos serían capaces de alcanzar. Por lo tanto, el proyecto pretende descubrir puntos comunes de referencia sobre las competencias y saberes de los egresados.

Buscando emular las experiencias europeas, Colombia es uno de los países que se ha propuesto formalmente construir su propio Marco Nacional de Cualificaciones (Ministerio de Educación de Colombia, 2017), cuyo propósito se enmarca en el contexto de ser el país más educado de América Latina a 2025. Por su parte, Perú, a través del Ministerio de Educación, y, con el apoyo de actores públicos y privados, busca desarrollar e implementar el MNC, cuyo propósito es el de contribuir a incrementar el nivel y relevancia de las cualificaciones de la población, enfocando dichos esfuerzos en el desarrollo y competitividad del país (Ministerio de Educación del Perú, 2019).

En Chile, una primera aproximación al desarrollo de un MNC surgió en el año 2007 en la cual las Universidades del CRUCH se adjudicaron el proyecto MECESUP UCN 701, donde se identificaron los elementos clave que debiese contener un MNC y los aspectos reglamentarios que deberían abordarse para su desarrollo. Luego, en 2010 Chile Califica, en conjunto con SEN$\mathrm{CE}$, intentó desarrollar un Marco de Cualificaciones, pero la iniciativa se vio descontinuada por el cierre del programa. En el año 2013 el Consejo Minero con apoyo de Fundación Chile, desarrollan el Marco de Cualificaciones para la Minería y en el 2014 Chile Valora, con respaldo del Banco Interamericano de desarrollo y la Organización Internacional del Trabajo, desarrollan el Marco de Cualificaciones para la formación y certificación laboral. En este contexto, el Consejo Nacional de Educación en el 2014 elaboró un documento titulado "Hacia un Marco Nacional de Cualificaciones para Chile", que define las razones para su construcción y cómo debiese ser llevado el proceso de desarrollo. El documento entrega públicamente al Ministerio de Educación, y específicamente a la División de Educación Superior, el desafío de elaborar el Marco Nacional 
de Cualificaciones para la Educación Superior (Kri et al., 2016). Finalmente, en 2016, se establece el Marco de Cualificaciones Técnico Profesional, cuyo objetivo "plantea asegurar oportunidades para el desarrollo de trayectorias laborales y formativas de estudiantes y trabajadores.", "aportando la construcción de un sistema de formación técnico profesional de calidad, pertinente a las necesidades de desarrollo local y económico, y articulado con las empresas e instituciones de formación" (Ministerio de Educación de Chile, 2016). Entre los principales objetivos del MNC Técnico Profesional, se encuentran:

1. Favorecer el desarrollo de un sistema de educación superior articulado, que permita el aprendizaje a lo largo de la vida y el reconocimiento de aprendizajes previos.

2. Establecer un sistema coherente, transparente y legible de certificaciones para la educación superior.

3. Promover la pertinencia de los perfiles de egreso de las carreras y programas en función de los requerimientos del medio social y laboral, y velar por su coherencia con el tiempo requerido para el logro de los aprendizajes correspondientes a cada uno de los niveles formativos.

Otros beneficios de contar con un MNC, incluyen reconocer y validar los aprendizajes obtenidos por las personas, facilitar la movilidad estudiantil y profesional entre IES, la internalización de una cultura de mejora continua y calidad educativa a nivel local, asegurando estándares mínimos internacionales, el mejor desarrollo de procesos de rediseño curricular orientados al logro de competencias, o la instalación de un Sistema de Créditos Transferibles en el sistema universitario (MECESUP, 2010). Algunas dificultades que persisten en Chile para su implementación son: una baja proporción de programas de pregrado acreditados, una legislación y normativa que en general pone el acento en la duración de los programas más que en los conocimientos, destrezas y competencias desarrollas en ellos, la falta de definición de estándares de calidad objetivos, y la baja articulación de las cualificaciones universitarias con el mundo del trabajo. A pesar de los esfuerzos indicados, aún no se ha definido o establecido un MNC integral para Chile, y tampoco se han hecho aplicaciones de estándares similares a la carrera de Contabilidad. El estudio más avanzado y sistemático es el trabajo coordinado por CINDA (2017) bajo el título "Evaluación del logro de perfiles de egreso: experiencias universitarias". En dicha investigación se recopilan múltiples estudios, diversos casos y diferentes aristas educativas. Por ejemplo, se rastrean diferentes enfoques metodológicos, marcos epistémicos y criterios de referencia para analizar los perfiles de egreso. Además, se reflexiona sobre diferentes experiencias internacionales tanto en América Latina como en Europa y se debate sobre la importancia de la evaluación en las IES. Por otro lado, se analiza la condición de egreso en diversas carreras estudiadas como ingeniería civil, educación y carreras ligadas al área de salud, pero no se ha analizado carreras de contabilidad.

Tomando como referencia los elementos descritos en torno a la implementación de MNC, la International Federation of Accountants (IFAC), mediante el Manual de Pronunciamientos Internacionales de Formación (PIF), establece el marco de referencia de los pronunciamientos de formación, en los que se menciona que "El desarrollo, la adopción y la implementación de los PIF proporcionan un método eficaz para el desarrollo de la competencia profesional". Otros resultados deseables incluyen:

- Reducir las diferencias internacionales en los requerimientos para desempeñar una función como profesional de la Contaduría; 
- Facilitar la movilidad global de los profesionales de la Contaduría; y

- Propiciar puntos de referencia internacionales de buenas prácticas para la formación profesional en Contaduría." (IFAC, 2015).

Por su parte, los autores, identificaron en un estudio previo las principales dificultades que afectan la inserción laboral de inmigrantes profesionales calificados en Chile tomando como ejemplo la carrera de contador auditor (Armijos y La Paz, 2019). En los años recientes, la cantidad de solicitudes de reconocimiento, convalidación y homologación de títulos universitarios obtenidos en el extranjero se incrementaron considerablemente, siendo la Universidad de Chile, la única institución facultada para realizar estos procesos. Otra dificultad es la cantidad de denominaciones que tiene la carrera, reportándose 39 distintas denominaciones en países de habla hispana en América Latina. La complejidad radica en que para cada denominación es necesario analizar y verificar la duración de la carrera (medida en semestres, ciclos, o módulos) y la composición y calidad de los programas. Un último elemento identificado, además de los requisitos formales, es la evaluación de criterios específicos, como el conocimiento de leyes y normas locales vigentes de calidad educativa, lo cual genera una muy baja tasa de reconocimiento y homologación de títulos extranjeros, revelando estos resultados la necesidad urgente de discutir y generar marcos que consoliden y unifiquen criterios para la formación contable en Latinoamérica y para la medición y acreditación de la calidad educativa.

Sumado a lo ya descrito, es interesante rescatar el aporte de Rodríguez, Gallegos, Palma y Rubilar (2019) cuyo estudio se enfoca en el perfil profesional del Contador Auditor, sobre las competencias técnicas de costos. El trabajo realiza un análisis descriptivo de los planes y programas de estudios de la carrera de Contador Público y Auditor de las universidades del Consejo de Rectores de Chile (CRUCH). Las competencias encontradas son muy amplias y van desde gestión de los costos, control de calidad, la medición de desempeño y comparaciones con estándares de referencia, a la planificación y diseño de presupuestos entre otras subespecialidades. Los resultados dan cuenta de las diferencias existentes en la formación de los auditores en cada una de las universidades, sin embargo, los contenidos mínimos requeridos por las empresas en Chile son cumplidos por todas las universidades. Se concluye, que al menos en lo que en materia de contabilidad gerencial se refiere, no existe homogeneidad en la enseñanza de los costos en la formación del Contador Público y Auditor, por lo cual, no se puede inferir que todos los contadores auditores en el mercado laboral cuenten con las mismas competencias técnicas. Es probable que estas diferencias sean también extensibles a la enseñanza de otras subdisciplinas componentes de los perfiles profesional y de egreso que cada universidad declara y difunde a la comunidad.

\section{METODOLOGÍA, MATERIALES Y MÉTODOS}

Los perfiles de carreras contables constituyen una definición institucional y un compromiso con la sociedad. Las IES diseñan e implementan programas de estudio y concretamente mallas curriculares para concretar la propuesta académica. Ambos elementos, perfil de egreso y malla curricular, presentan una amplia variedad de competencias, habilidades a desarrollar, saberes y cursos entre los que se debe elegir y decidir si el programa enfatizará algún enfoque en particular o pretenderá lograr un profesional generalista. Para poner toda esta información en contexto de manera ordenada y sistemática, se ha construido un marco ontológico, desarrollado en el marco 
de un programa de investigación de tipo cualitativo-exploratorio, que permite describir y visibilizar de manera integral los elementos y componentes disponibles en los procesos de diseño y construcción curricular, así como analizar sistemáticamente los constructos académicos implícitos en las carreras que actualmente se dictan en Chile, notando explícitamente los elementos presentes y ausentes en los perfiles y mallas curriculares. El marco ontológico conceptualiza los dominios de desempeño profesional de manera exhaustiva, revelando a través de la organización y estandarización de términos en taxonomías, las posibles combinaciones que finalmente se expresan en un perfil y malla curricular, o elementos específicos de la ontología podrían quedar contenidos en syllabus y programas de curso. La construcción de marcos ontológicos ha resultado un instrumento de gran utilidad, siendo un método aplicado en varios estudios de Educación Superior (Ramaprasad 2011; Coronado et al 2015; La Paz y Arrúa, 2019), Sistemas de Información (Vásquez y La Paz, 2019; La Paz et al, 2020), Gestión en salud (Núñez, Ramaprasad, 2015), por citar algunos.

La Figura 1 muestra el marco ontológico correspondiente a las carreras de contabilidad, junto con ejemplos de combinaciones de términos que frecuentemente se explicitan en perfiles profesionales o en descripciones de cursos en las mallas curriculares. Al respecto, el marco ontológico incluye las taxonomías que representan a) Objetivo educativo, b) Competencias, c) Productos y d) Dominio de desempeño. Cada taxonomía se construye a partir de referencias académicas, recopilaciones del medio académico y profesional, y en este caso particular, desde la revisión de perfiles de egreso y mallas curriculares de una amplia muestra de carreras de contabilidad, con el objetivo de que cada taxonomía sea completa. Las taxonomías (en columnas) se organizan de manera tal que al combinar secuencias de elementos de cada una y utilizando conectores intermedios, se forman frases que frecuentemente vemos contenidas en perfiles de egreso y perfiles profesionales, lo que permite clasificar sistemáticamente los elementos de cada perfil y malla curricular para descubrir los énfasis u omisiones comprometidas por cada IES. En particular, este estudio cubre el universo de carreras universitarias de contabilidad que ofrecen IES chilenas. 


\section{Figura 1}

Marco Ontológico para carreras de Contabilidad.

\begin{tabular}{|c|c|c|c|}
\hline $\begin{array}{c}\text { Objetivo } \\
\text { Educativo }\end{array}$ & Competencias & Productos & Dominio \\
\hline $\begin{array}{l}\text { Recordar } \\
\text { Comprender } \\
\text { Aplicar } \\
\text { Analizar } \\
\text { Evaluar } \\
\text { Crear }\end{array}$ & $\begin{array}{l}\text { Técnicas para } \\
\text {.Asesorar } \\
\text {. Auditar } \\
\text {. Emitir opinión } \\
\text {. Diagnosticar } \\
\text {. Interpretar } \\
\text {. Diseñar } \\
\text {. Toma de decisiones } \\
\text {. Optimizar recursos } \\
\text {. Actualización profesional } \\
\text {. Implementar } \\
\text {.Planificar } \\
\text {. Construir/Elaborar } \\
\text { Transversales para } \\
\text {.Emprender e innovar } \\
\text {. Liderar } \\
\text {.Compromiso social } \\
\text {. Segundo idioma } \\
\text {.Comunicación efectiva } \\
\text {. Trabajo en equipo } \\
\text {.Uso de recursos de información } \\
\text {. Aprendizaje autónomo } \\
\text {. Motivación y adaptación } \\
\text {.Ética } \\
\text {. Confidencialidad } \\
\text {. Integridad } \\
\text {. Objetividad } \\
\text {. Relaciones interpersonales } \\
\text {. Razonamiento crítico y } \\
\text { resolución de problemas }\end{array}$ & $\begin{array}{l}\text { Sistemas } \\
\text { Controles } \\
\text { Normas } \\
\text { Proyectos } \\
\text { Procesos } \\
\text { Servicios } \\
\text { Informes y reportes }\end{array}$ & $\begin{array}{l}\text { Administración y operaciones } \\
\text { Auditoría } \\
\text { Contabilidad } \\
\text { Economía } \\
\text { Métodos cuantitativos } \\
\text { Finanzas } \\
\text { Legal } \\
\text { Sistemas de Información } \\
\text { Formación complementaria }\end{array}$ \\
\hline
\end{tabular}

\section{Ejemplos}

* Analizar habilidades transversales para emprender e innovar en proyectos de sistemas de información

* Crear habilidades técnicas para actualización profesional en procesos de auditoría

* Evaluar habilidades técnicas para diagnosticar procesos en finanzas

* Aplicar habilidades técnicas para Optimizar recursos en proyectos de auditoría

* Crear habilidades transversales para emprender e innovar en controles del área Legal

* Recordar habilidades transversales para segundo idioma en informes y reportes en contabilidad

Nota: La figura muestra el marco ontológico para las carreras de contabilidad, tomando elementos del perfil de egreso (Objetivo educativo, Competencias y Productos), y Malla Curricular (Dominio). Elaboración Propia.

La recolección de datos se realizó entre febrero y junio de 2019 indagando cada una de las 56 universidades vigentes en Chile para verificar cuántas de ellas ofrecen una carrera del área contabilidad, así como registrar los datos de su denominación, perfil de egreso y malla curricular. Este estudio analiza las carreras de contabilidad que tengan una duración mínima de 8 semestres y cuenten con información pública de perfil de egreso y malla curricular. La Tabla 1 resume cuáles son los datos incorporados al estudio. De un total de 56 universidades, 36 instituciones ofrecen grados de licenciatura y títulos profesionales en contabilidad y / o auditoría. 


\section{Tabla 1}

\section{Lista de Universidades que dictan la carrera de Contador Auditor en Chile.}

\begin{tabular}{|c|c|c|}
\hline $\mathrm{N}^{\circ}$ & Universidad & Denominación del Programa \\
\hline 01 & Universidad de Chile & Contador Auditor \\
\hline 02 & Pontificia Universidad Católica de Valparaíso & Contador Auditor \\
\hline 03 & Universidad Católica de Temuco & Contador Auditor \\
\hline 04 & Universidad Andrés Bello & Contador Auditor \\
\hline 05 & Escuela de Contadores Auditores de Santiago & Contador Auditor \\
\hline 06 & Universidad Católica de la Santísima Concepción & Contador Auditor \\
\hline 07 & Universidad de las Américas & Contador Auditor \\
\hline 08 & Universidad de Viña del Mar & Contador Auditor \\
\hline 09 & Universidad de Artes, Ciencias y Comunicación & Contador Auditor \\
\hline 10 & Universidad Adventista de Chile & Contador Auditor \\
\hline 11 & Universidad Bernardo O’Higgins & Contador Auditor \\
\hline 12 & Universidad de Valparaíso & Contador Auditor \\
\hline 13 & Universidad Católica del Maule & Contador Auditor \\
\hline 14 & Universidad Miguel de Cervantes & Contador Auditor \\
\hline 15 & Universidad Autónoma de Chile & Contador Auditor \\
\hline 16 & Universidad Finis Terrae & Contador Auditor \\
\hline 17 & Universidad Central de Chile & Contador Auditor \\
\hline 18 & Universidad de Concepción & Contador Auditor \\
\hline 19 & Universidad Austral de Chile & Contador Auditor \\
\hline 20 & Universidad Tecnológica de Chile INACAP & Contador Auditor \\
\hline 21 & Universidad de Santiago de Chile & Contador Público y Auditor \\
\hline 21 & Universidad de Talca & Contador Público y Auditor \\
\hline 22 & Universidad del Bío Bío & Contador Público y Auditor \\
\hline 24 & Universidad Arturo Prat & Contador Público y Auditor \\
\hline 25 & Universidad Santo Tomás & Contador Público y Auditor \\
\hline 26 & Universidad Católica Cardenal Raúl Silva Henríquez & Contador Público y Auditor \\
\hline 27 & Universidad Tecnológica Metropolitana & Contador Público y Auditor \\
\hline 28 & Universidad de Los Lagos & Contador Público y Auditor \\
\hline 29 & Universidad Mayor & Contador Público y Auditor \\
\hline 30 & Universidad de Tarapacá & Contador Auditor-Contador Público \\
\hline 31 & Universidad Católica del Norte & Contador Auditor-Contador Público \\
\hline 32 & Universidad de Magallanes & Contador Auditor-Contador Público \\
\hline 33 & Universidad Alberto Hurtado & Contador Público Auditor \\
\hline 34 & Universidad de la Serena & Contador Público Auditor \\
\hline 35 & Universidad de la Frontera & Contador Público - Contador Auditor \\
\hline 36 & Universidad Diego Portales & Auditor - Contador Público \\
\hline
\end{tabular}

Fuente: Subsecretaría de Educación Superior (2020). 
Los programas fueron clasificados en el marco ontológico de contabilidad en dos etapas. La primera utiliza las definiciones de perfil de egreso (en algunos casos, complementado con objetivos de la carrera, actuación profesional, campo laboral, habilidades, conforme a la información publicada por cada institución), y clasifica manualmente el contenido de estos textos en los elementos de las primeras tres columnas del marco ontológico (Objetivos educativos, Competencias, y, Productos), indicando en cada caso, la presencia (1) o ausencia (0) de los términos en las taxonomías, en los perfiles de egreso publicados. La segunda etapa, también clasifica manualmente el contenido de las mallas curriculares, esto es, cada curso ofrecido en el programa, en los dominios que la columna final del marco ontológico describe. En los resultados se mostrarán las frecuencias con que las universidades se comprometen con la formación de los elementos en el perfil de egreso, y el promedio de los cursos ofrecidos en los dominios correspondientes a las carreras de contabilidad.

\section{RESULTADOS}

De acuerdo con el análisis de los perfiles de egreso que cada universidad presenta, se muestra a grandes rasgos una heterogeneidad y diversidad en las competencias que los egresados desarrollan. Otro elemento por mencionar es el referente a la cantidad de denominaciones que la carrera ha adquirido en Chile. De las 36 universidades que ofrecen la carrera universitaria de Contabilidad, se presentan 6 denominaciones distintas. Entre ellas: Contador Auditor: 20 universidades, Contador Público y Auditor: 9 universidades, Contador Auditor - Contador Público: 3 universidades, Contador Público Auditor: 2 universidades, Contador Público - Contador Auditor: 1 universidad, y, Auditor - Contador Público: 1 universidad. Al respecto, no se ha logrado identificar el motivo de la diferenciación en las denominaciones. En cuanto a la estructura de las carreras, no se aprecia un nivel de diferenciación que permita establecer un criterio que pueda establecer una relación significativa con las distintas denominaciones.

El marco ontológico presenta cuatro categorías diferentes para analizar los perfiles de egreso: Productos, Competencias Transversales, Competencias Técnicas, y Objetivos Educacionales. Junto a cada elemento en las taxonomías, se reporta la frecuencia de aparición de estos términos (o su sinónimo) en la muestra correspondiente al universo de las 36 carreras universitarias de contabilidad en Chile.

En cuanto a los Productos que se declara que un egresado puede ofrecer al mercado, según los perfiles de egreso, sobresalen por su frecuencia encontrada en la población de carreras de contabilidad Servicios (19), Sistemas (18), e Informes y Reportes (17). Por otra parte, en materia de Competencias Transversales definidas en el diseño curricular y declaradas en los perfiles de egreso publicados por cada universidad, la Ética (24) como Emprender e Innovar (23), además del Uso de Recursos de Información (15), el Compromiso Social (15) y el Trabajo en Equipo (18). Con respecto a las Competencias Técnicas, las universidades declaran que sus egresados son capaces de Asesorar (24), Auditar (19) y Diseñar (19), aunque también puede encontrarse Emitir Opinión (16), Diagnosticar (13), Planificar (9) y otras habilidades. Por último, los principales objetivos educativos en los currículos de las IES en Chile son: Crear (22), Analizar (19) y Evaluar (17). 
Por otra parte, en cuanto a la malla curricular, se hace referencia al dominio de materias que dispone el egresado respecto de las carreras de contabilidad. En este dominio, se reporta entre paréntesis el promedio de cursos contenido en las mallas curriculares, y aunque no es exactamente comparable con la frecuencia de universidades que declaran objetivos educativos, competencias y productos en sus perfiles de egreso, es interesante contrastar la "promesa" establecida en los perfiles de egreso, con la "ejecución" de una construcción de malla curricular. Como es posible apreciar, entre una vasta lista de posibilidades sobresalen la Auditoría $(4,8)$, la Contabilidad $(8,7)$, y la Administración y Operaciones (6,3).

Es importante destacar el bajo número promedio de cursos relacionados con Sistemas de Información $(3,8)$, en relación con la alta presencia de cursos referentes al Formación Complementaria $(15,0)$ que incluye, por ejemplo, cursos deportivos, idiomas, o formación de habilidades interpersonales. Por otra parte, llama la atención verificar que cursos relacionados con la Ética $(0,8)$, estén presentes en menor proporción que aquellos cursos relacionados con Idiomas $(3,6)$, en general referidos a inglés. Sin duda, ambos son importantes en la formación de cualquier profesional; pero en la formación de la ética profesional, se establecen los principios que norman la conducta del contador auditor, lo que sí es enfatizado en los perfiles de egreso.

El marco ontológico permite también visibilizar las brechas que, en este caso, las universidades chilenas plasman por omisión en los perfiles de egreso y mallas curriculares. Una omisión positiva se relaciona con el objetivo educativo de Recordar, que no es declarado por ninguna universidad en el perfil de egreso. Lo positivo de esta omisión del nivel más básico entre los objetivos educativos, podría atribuirse a la transición hacia programas por competencias que han logrado instalar una aspiración a objetivos educativos de mayor complejidad. Otras omisiones o brechas importantes para notar en los perfiles son actualización profesional, optimización de recursos, motivación y adaptación, relaciones interpersonales, y desarrollo de proyectos. Mayor detalle de frecuencia de universidades que declaran compromisos en perfiles de egreso, y promedio de cursos en las mallas curriculares, se aprecian en la Figura 2. 


\section{Figura 2}

\section{Mapa ontológico de las carreras de contabilidad en Chile.}

\begin{tabular}{|c|c|c|c|c|c|c|}
\hline Objetivo Edu & & Competencias profesional & & Productos & Dominio & \\
\hline Recordar & & Asesorar & & Sistemas & Administración y operacione & \\
\hline Compronder. & 0 & 1. & 24 & 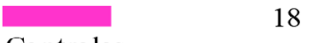 & 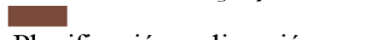 & $\begin{array}{c}6,3 \\
(4,2)\end{array}$ \\
\hline Comprender & 1 & Auditar & & Controles & $\begin{array}{l}\text { Planificación y dirección } \\
\text { RRHH }\end{array}$ & $\begin{array}{l}(4,2) \\
(0,9)\end{array}$ \\
\hline Aplicar & & Emitir opinión & & Informes y/o reportes & Marketing/mercadeo & $(0,4)$ \\
\hline & 9 & & 16 & 17 & Procesos de negocio & $(0,8)$ \\
\hline Analizar & & Diagnosticar & & Normas & Auditoría & \\
\hline & 19 & (1) & 13 & 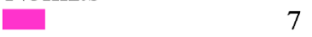 & $\square$ & 4,8 \\
\hline Evaluar & & Interpretar & & Proyectos & Auditoría & $(2,1)$ \\
\hline$\square$ & 17 & & 12 & - & Control interno & $(0,5)$ \\
\hline Crear & & Diseñar & & Procesos & Estados Financieros & $(1,3)$ \\
\hline & & 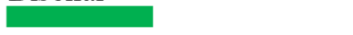 & 19 & $\square$ & Forense & $(0,1)$ \\
\hline & & Tomar de decisiones & & Servicios & Procesos & $(0,2)$ \\
\hline & & a & 14 & 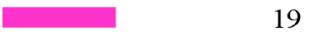 & Riesgos & $(0,0)$ \\
\hline & & Optimizar recurs os & 1 & & $\begin{array}{l}\text { Tecnológica } \\
\text { Contabilidad }\end{array}$ & $(0,7)$ \\
\hline & & Actualización profes ional & & & & 8,7 \\
\hline & & & 1 & & General & $(4,1)$ \\
\hline & & Implementar & & & Tributaria & $(2,0)$ \\
\hline & & & 9 & & Financiera & $(0,8)$ \\
\hline & & Planificar & & & Gestión & $(0,3)$ \\
\hline & & & 9 & & Costos y Presupuestos & $(3,1)$ \\
\hline & & Construir & & & Economía & \\
\hline & & & 5 & & $\square$ & 2,8 \\
\hline & & & & & Micro & $(1,5)$ \\
\hline & & & & & Macro & $(1,2)$ \\
\hline & & Emprender e innovar & & & Sustentable & $(0,03)$ \\
\hline & & - the & 23 & & Política & $(0,1)$ \\
\hline & & Liderar & & & Métodos cuantitativos & \\
\hline & & & 15 & & 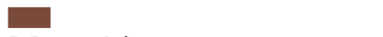 & 4,5 \\
\hline & & Compromiso social & & & Matemática & $(3,2)$ \\
\hline & & & 15 & & Estadística & $(1,3)$ \\
\hline & & Segundo idioma & & & Instrumental aplicado & $(0,1)$ \\
\hline & & (1) & 7 & & Finanzas & \\
\hline & & Comunicación efectiva & & & 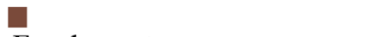 & $\mathbf{2 , 1}$ \\
\hline & & & 9 & & Fundamentos & $(1,4)$ \\
\hline & & Trabajo en equipo & & & Personales & $(0,0)$ \\
\hline & & $x^{2}$ & 18 & & Corporativas & $(0,5)$ \\
\hline & 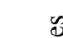 & Uso de recursos de Info. & & & Públicas & $(0,2)$ \\
\hline & 跑 & 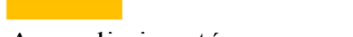 & 15 & & Legal & \\
\hline & 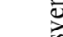 & Aprendizaje autónomo & 2 & & & $\begin{array}{l}3,6 \\
(1)\end{array}$ \\
\hline & & R. crítico y res. de problema & $s^{3}$ & & $\begin{array}{l}\text { Comercial } \\
\text { Tributaria }\end{array}$ & $\begin{array}{l}(1,1) \\
(1,4)\end{array}$ \\
\hline & 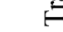 & & 8 & & Laboral & $(0,7)$ \\
\hline & & Motivación y adaptación & & & Civil & $(0,3)$ \\
\hline & & & 0 & & Sistemas de Información & \\
\hline & & Élica & & & $\square$ & 3,8 \\
\hline & & 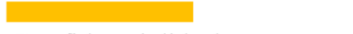 & 24 & & Sistemas Info. Admin. & $(2,1)$ \\
\hline & & Confidencialidad & & & Bases de datos & $(0,2)$ \\
\hline & & & 6 & & Anális is de datos & $(0,3)$ \\
\hline & & Integridad & & & Infraestructura & $(0,7)$ \\
\hline & & & 8 & & Aplicaciones (SW) & $(0,3)$ \\
\hline & & Objetividad & & & Programación & $(0,1)$ \\
\hline & & (5) & 11 & & Gestión de proyectos & $(0,1)$ \\
\hline & & Relaciones interpersonales & & & Formación complementaria & \\
\hline & & & 1 & & & 15,0 \\
\hline & & & & & Idiomas & $(3,6)$ \\
\hline & & & & & Etica & $(0,8)$ \\
\hline & & & & & Métodos de investigación & $(0,3)$ \\
\hline & & & & & Habilidades Interpersonales & $(1,4)$ \\
\hline & & & & & Deptes y formación Integral & $(0,6)$ \\
\hline & & & & & Práctica profesional & $(1,6)$ \\
\hline & & & & & Otros & $(6,7)$ \\
\hline
\end{tabular}

Nota: La Figura muestra los resultados de la aplicación del marco ontológico. Los Perfiles de Egreso están conformados por el objetivo educativo, las competencias profesionales y los productos. Mientras que, las mallas curriculares por el dominio. Elaborado por los autores. 
A partir de los resultados, podemos inferir que algunos de los compromisos formativos más comunes para la carrera de contabilidad en Chile son:

- Crear habilidades para asesorar, auditar y diseñar informes y reportes

- Formar habilidades para analizar sistemas de control y emitir una opinión profesional

- Evaluar la ética en el uso de los recursos de información

Algunas omisiones, o menores énfasis plasmados en los compromisos formativos son:

- Comprender la optimización de recursos en proyectos

- Aplicar habilidades para la actualización profesional en las normas

- Recordar habilidades para establecer relaciones interpersonales en procesos

\section{DISCUSIÓN Y CONCLUSIONES}

Ante exigencia progresiva en la formalización de mecanismos y procesos de evaluación y certificación de la calidad establecidos por la CNA y el modelo de evaluación y certificación de la calidad educativa, las universidades han respondido a esta motivación con actualizaciones, mejoras y / o reformulaciones de sus programas académicos, buscando a la vez diferenciarse en un medio competitivo mediante la presentación de perfiles de egreso, denominaciones de programas y construcción curricular. La falta de algún marco de referencia, como los marcos de cualificaciones, puede generar un sentido de libertad de cátedra para que cada institución educativa detecte necesidades del medio y proponga programas que resuelvan problemas reales, pero por otra parte deja espacios importantes en desorganización que pueden llevar a confusión en el ámbito universitario (postulantes, estudiantes, docentes, directivos) y laboral (egresados, empleadores). Lo anterior se produce a nivel local, pero internacionalmente se dificulta aún más el intercambio académico y la movilidad de capital humano.

El estudio refleja la complejidad de la situación ya que en Chile existe una variedad de nombres que la carrera de contabilidad adquiere, registrándose seis diferentes. Aunque ha sido planteado como un objetivo del estudio detectar las diferencias asociadas a las denominaciones, el tamaño reducido de la población total de programas ofrecidos en Chile no permitió revelar diferencias significativas y confiables para reportar, por lo que será abordado con una muestra internacional de acuerdo al programa de investigación que los autores promueven. A su vez, cada universidad, a través del complejo proceso del diseño curricular, propone diferentes características en el perfil de egreso, sobre lo cual este estudio logra resumir los énfasis u omisiones que las universidades chilenas ofrecen a postulantes así como al mercado laboral respecto de los saberes, competencias y desempeño esperado. Es importante en este último punto insistir en que hay dominios del conocimiento que el mercado considera relevantes y en Chile son poco o nada valorados como por ejemplo la inteligencia o analítica de negocios, ges- 
tión de riesgos, auditoría forense, análisis de datos, o economía política y economía sustentable. Lo mismo sucede con competencias técnicas, tales como optimizar recursos y la actualización profesional. La ética es un compromiso formativo expresado en los perfiles de egreso por dos tercios de las universidades que ofrecen la carrera, sin embargo, el número promedio de cursos encontrados en todas las mallas curriculares es inferior a uno. Una posible interpretación de esta inconsistencia podría ser que la formación ética no se desarrolla en un solo curso o actividad formativa, sino que se trabaja en varios cursos, aplicando por ejemplo el estudio de dilemas éticos en temas de impuestos, contabilidad, auditoría, finanzas, control interno o administración en general. También existe la posibilidad de una nula formación académica de esta competencia esencial en el contador auditor, lo que reflejaría una mayor inconsistencia entre lo declarado en un perfil de egreso, y lo ejecutado en la malla curricular.

Este análisis abre nuevas interrogantes, por ejemplo, en definir si las IES declaran en el perfil de egreso lo que realmente ejecutan en los cursos definidos en sus mallas curriculares, dando transparencia al público y siendo consistentes con su compromiso. El caso óptimo sería que se declaran y ejecutan los programas que construyen las competencias y desempeños que compromete el perfil de egreso, sin embargo, no ignoramos la posible existencia de perfiles y mallas que no son completamente coherentes, o incluso a la ejecución curricular de mallas que son más complejas y completas de lo que se declara en un perfil de egreso. Cualquiera sea el caso, los autores recomiendan evaluar y ajustar los elementos comprometidos declarados y ejecutados, de manera de lograr coherencia y consistencia educativa, como se observa críticamente durante los procesos de autoevaluación, certificación y acreditación de calidad educativa.

De los resultados se observa una gran inclinación del contador auditor en Chile hacia las áreas de Administración y Operaciones, Contabilidad, Métodos Cuantitativos, y en proporción importante hacia las áreas de Formación Complementaria. Sin embargo, se nota un menor interés sobre las áreas de sistemas de información, siendo estas de gran importancia, dado que el contador auditor se desenvuelve en buena parte de su actividad laboral con apoyo de tecnología y herramientas informáticas para la generación, respaldo, procesamiento y transferencia de información.

Finalmente, se aprecia que la construcción curricular de las carreras de Contabilidad en Chile oscila entre dos modelos: uno generalista, vale decir, una formación con conocimientos básicos de administración; y el de planes de estudio con conocimientos específicos, esto es, especialistas en determinada área de la carrera. La definición de un perfil y construcción generalista o uno especialista es un desafío mayor, ya que en base a ello, la construcción de competencias y habilidades establecidas en perfiles de egreso y programas curriculares deben ser consistentes con el propósito formativo, y contemplar además aquellos que el mercado laboral considera necesarios para este profesional. Por ejemplo, un perfil generalista debería incluir y enfatizar la capacidad de actualizarse profesionalmente, mientras que un perfil especialista podría promover y vincular un programa de pregrado con uno de postgrado en sub-disciplinas de la profesión. 


\section{REFERENCIAS BIBLIOGRAFICAS}

Armijos, J. y La Paz, A. (2019) Dificultades para la movilidad internacional del contador auditor. Revista Contabilidad y Sistemas, Vol 17, pp. 33-44.

CEDEFOP. (2012). Marcos de Cualificaciones en Europa: Un instrumento para la transparencia y el cambio. Nota Informativa. Octubre 2012. ISSN 1831-2446.

CEDEFOP. (2017). Marcos de Cualificaciones en Europa. Evoluciones 2017. Nota Informativa. Febrero 2018. ISSN 1831-2446.

CINDA. (2017). Evaluación del logro de perfiles de egreso: experiencias universitarias. Centro Interuniversitario de Desarrollo. Grupo Operativo de Universidades Coordinadas por CINDA. Santiago de Chile.

Coronado, F., La Paz, A., Ramaprasad, A., \& Syn, T. (2015). Navigating the Complexity and Uncertainty of Higher Education Systems: Ontology Mapping of Chile's Universities. Proceedings of HERDSA 2015, Melbourne, Australia.

International Accounting Education Standards Board. (2015). Manual de Procedimientos Internacionales de Formación. Recuperado el 17 de noviembre de 2020, https: / / www.ifac.org/ system / files / publications / files / Manual-de-Pronunciamientos-Internacionales-de-Formacion-Edicion-2015.pdf

International Federation of Accountants. (2019). International Accounting Education Standards Board. Handbook of International Education Pronouncements. Recuperado el 12 de enero de 2021, https://www.ifac.org/system/files/publications / files/Handbook-of-International-Education-Standards-2019.pdf

Kri, A. F., Marchant, M. E., Lazo, L. M., \& Cruz, F. S. (2016). Marco Nacional de Cualificaciones para la Educación Superior. Santiago de Chile, Ministerio de Educación.

La Paz, A., \& Arrúa, K. (2019). Mapa de las Aspiraciones de la Educación Superior en Paraguay. Revista del CLAD Reforma y Democracia, 74 (Junio).

La Paz, A., Merigó, J. M., Powell, P., Ramaprasad, A., \& Syn, T. (2020). Twenty- five years of the Information Systems Journal: A bibliometric and ontological overview. Information Systems Journal, 30(3), 431-457.

López, R., (2016) Gestión estratégica de proyectos de desarrollo en sistemas de información: aplicación en la gestión universitaria. Tesis para optar al grado de Magíster en Control de Gestión, Universidad de Chile.

López-Peña, A. (2017). Características de los procesos de Innovación Curricular en las Instituciones de Educación Superior de Chile. Tesis para optar al grado de Magíster en Educación con Mención en Currículo y Comunidad Educativa.

MECESUP. (2010). Diseño de un Marco de Cualificaciones para el Sistema de Educación Superior Chileno. Hacia un Sistema coherente y legible de educación superior. Santiago, Chile. Proyecto MECESUP UCN 0701.

Ministerio de Educación de Chile. (2016). Marco de Cualificaciones Técnico Profesional. Hacia un Marco de Cualificaciones Técnico Profesional. Santiago, Chile.

Ministerio de Educación de Colombia. (2017). Marco Nacional de Cualificaciones. Un camino para la inclusión, la equidad y el reconocimiento. Gobierno de Colombia. Recuperado de https:/ / www.mineducacion.gov.co/1759/articles-362828_recurso.pdf

Ministerio de Educación del Perú. (2019). Minedu diseña el nuevo Marco Nacional de Cualificaciones [Comunicado de prensa]. Recuperado de https: / / www.gob.pe/institucion/minedu / noticias / 68671-minedu-disena-el-nuevo-marco-nacional-de-cualificaciones 
Nuñez-Mondaca, A., Ramaprasad, A., \& Syn, T. (2015). National Healthcare Policies in Chile: An Ontological Meta-Analysis. Medinfo. p. 1105.

Ramaprasad, A. (2011). Envisioning a world-class university system for India. International Journal of Technology Management \& Sustainable Development, 10(1), pp. 45-54.

Rodríguez, E. Gallegos, C. Palma, I. Rubilar, A. (2019). Competencias técnicas de costos en el perfil profesional del Contador Auditor Chileno. Revista Venezolana de Gerencia, vol. 24, núm. 86, 2019.

Troncoso, K., y Hawes, G. (2007). Taller de construcción curricular. Acuerdos básicos sobre el perfil de egreso profesional (pp. 4). Santiago: Universidad de Chile, Vicerrectoría de Asuntos Académicos, Departamento de Pregrado.

Vásquez, Jonathan, \& La Paz, Ariel. (2019). The Knowledge Body of Requirement Engineering in IST Innovations: An Ontological Analysis. Journal of technology management \& innovation, 14(4), 78-84.

Wyman, Harold, E. (1990). El poder y la Importancia de la Contabilidad. Ayer, hoy y mañana; cómo fue, cómo es y cómo debería ser. Revista Española de Financiación y Contabilidad, Vol. $X X, N^{\circ} .62$, pp. 9-25.

Zúñiga, F. V. (2018). Avanzando hacia los Marcos Nacionales de Cualificaciones: la experiencia de Chile y República Dominicana. Montevideo: OIT/Cinterfor, 2018.94p. ISBN: 978-92-9088290-9

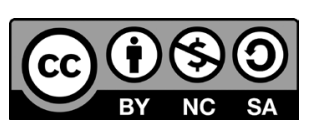

Esta obra está bajo una licencia de

Creative Commons Atribución-NoComercial-CompartirIgual 4.0 Internacional 